\title{
MicroRNA-mRNA integrated analysis based on a case of well-differentiated thyroid cancer with both metastasis and metastatic recurrence
}

\author{
YUN-JIE ZHANG $^{1 *}$, YU-SHUI MA ${ }^{2,3^{*}}$, QING XIA ${ }^{1 *}$, FEI YU ${ }^{3}$, ZHONG-WEI LV ${ }^{3}$, CHENG-YOU JIA ${ }^{3}$, \\ XIAO-XIN JIANG ${ }^{1}$, LIANG ZHANG ${ }^{1}$, YUN-CHAO SHAO ${ }^{1}$, WEN-TING XIE ${ }^{3}$, GAI-XIA LU ${ }^{3}$, \\ XIA-QING $\mathrm{YV}^{3}$, PENG ZHONG ${ }^{3}, \mathrm{DA} \mathrm{FU}^{4}$ and XIAO-FENG WANG ${ }^{1}$ \\ ${ }^{1}$ Department of Orthopedics, Zhongshan Hospital, Fudan University, Shanghai 200032; \\ ${ }^{2}$ Shanghai Engineering Research Center of Molecular Therapeutics and New Drug Development, \\ College of Chemistry and Molecular Engineering, East China Normal University, Shanghai 200062; \\ ${ }^{3}$ Department of Nuclear Medicine and ${ }^{4}$ Central Laboratory for Medical Research, Shanghai Tenth People's Hospital, \\ Tongji University School of Medicine, Shanghai 200072, P.R. China
}

Received April 17, 2018; Accepted September 19, 2018

DOI: $10.3892 / o r .2018 .6739$

\begin{abstract}
The incidence of well-differentiated thyroid cancer (WDTC) is rapidly increasing. Poor survival follows distant metastasis (DM) and recurrence. In the present study, we aimed to analyze the expression alterations in different stages of WDTC and the regulatory mechanism of DM and the recurrence of DM. A male patient diagnosed with follicular thyroid cancer and distant metastasis in the eleventh thoracic vertebrae received total thyroidectomy and the removal of a metastatic lesion. A local relapse was found in the vertebrae after four-time iodine-131 treatment. We performed mRNA and microRNA microarray on the paracancerous, cancerous, metastatic and metastatic recurrent tissue. In combination with the data of The Cancer Genome Atlas (TCGA), we used bioinformatics approaches to analyze the common alterations and microRNA-mRNA interactions among the processes of tumorigenesis and metastasis. Metastatic lesions and recurrent lesions were used to investigate the molecular mechanism of tumor evolution and recurrence in this case. A total of four mRNAs and two microRNAs were newly found to be
\end{abstract}

Correspondence to: Dr Xiao-Feng Wang, Department of Orthopedics, Zhongshan Hospital, Fudan University, 180 Fenglin Road, Shanghai 200032, P.R. China

E-mail:wxf2002@hotmail.com

Professor Da Fu, Central Laboratory for Medical Research, Shanghai Tenth People's Hospital, Tongji University School of Medicine, Middle 301 Yanchang Road, Shanghai 200072, P.R. China E-mail: fu800da900@126.com

*Contributed equally

Key words: WDTC, metastasis, recurrence, microarray, miRNAmRNA interaction related to patient survival in WDTC. The microRNA-mRNA interactions were predicted for the overlapped mRNAs and microRNAs. Lineage deregulation of genes, such as C-X-C motif chemokine receptor 4 (CXCR4) and thyroglobulin (TG) were found from the tumorigenic stage to the metastatic stage. The ribosome pathway was highly enriched in the bone metastasis compared with the cancerous tissue. The downstreaming effects of p53 were impaired in the recurrent lesion due to deregulation of several functional genes. The integrated analysis with TCGA data indicated several prognostic markers and regulatory networks for potential treatment. Our results also provided possible molecular mechanisms in which the ribosome and p53 pathways may respectively contribute to bone metastasis and local recurrence of metastasis

\section{Introduction}

Thyroid cancer, the most common endocrine malignancy worldwide, has increased 3 -fold in the past 3 decades $(1,2)$. Well-differentiated thyroid cancer (WDTC), including papillary and follicular thyroid cancer, represents $>90 \%$ of all thyroid cancers (3), and patients with WDTC have a 10-year survival of $80-95 \%$. Many studies have profiled WDTC genes and rearrangements of $R E T / P T C$ and $P A X 8 / P P A R \gamma$ (4), and frequent (70\%) point mutations of $B R A F$ and $R A S$ genes, which alter the mitogen-activated protein kinase (MAPK) signaling pathway (5-7) were documented in WDTC. Several genes, such as $M U C 1, P D-L 1, D P P 4$, and mutations of the $B R A F^{V 600 E}$ and $T E R T$ promoter, have been reported as prognostic markers (8-11).

For WDTC, 10-year survival is reduced to $14-40 \%$ when distant metastasis (DM) occurs (12-14). For patients with metastases, radioactive iodine $\left({ }^{131} \mathrm{I}\right)$ has been the mainstay of treatment, selectively combined with surgical intervention, external beam radiation and chemotherapy (15). Metastatic lesions are sometimes resistant to ${ }^{131} \mathrm{I}$, so treatment options are limited and survival is poor (10-year survival, 10\%) $(14,16)$. 
Recurrence also greatly contributes to the morbidity of WDTC $(17,18)$, and recurrence at 30 years is $35 \%$, most being local with $32 \%$ being DM (17). Thus, we must identify potential targets to improve therapeutic strategies to prevent metastasis and recurrence.

MicroRNAs (miRNAs) are a class of endogenous small (18-22 nucleotide) non-coding RNAs. Gene expression regulation by miRNAs is of interest because many miRNAs in WDTC, such as miR-221, miR-222 and miR-146 (19-21), have been reported to have critical roles in the regulation of apoptosis, proliferation, the cell cycle and epithelial-mesenchymal transition by negatively regulating mRNAs post-transcriptionally. Other studies on mRNA and miRNA expression indicate a miRNA-mRNA regulatory network that may provide clues for genetic deregulation in WDTC $(22,23)$. Although many studies have focused on the tumorigenesis of WDTC, few studies have identified the mechanism of metastasis and recurrence in WDTC. This is due to the fact that tissues must be taken from metastatic sites, yet metastasis-prone sites of WDTC, such as the brain and bones, are challenging to sample properly. Second, the incidence of metastasis and recurrence is relatively low, and as such, WDTC patients have better outcomes than other malignancies.

In the present study, we collected tissue samples from four separate stages of WDTC: Normal, cancerous, metastatic and recurrent stages and we performed mRNA and miRNA microarray analysis. By integrating our study with gene expression data from The Cancer Genome Atlas (TCGA) (21), we revealed novel prognostic markers and key genes related to the progression of WDTC and possible pathways involved in WDTC recurrence.

\section{Materials and methods}

Pipeline and workflow. We observed changes in the expression of mRNA and miRNA in normal, cancerous, metastatic and recurrent stages of WDTC. To study tumorigenesis and metastasis, we overlapped our results with differentially expressed mRNAs (DEmRNAs) and miRNAs (DEmiRNAs) from TCGA sequencing data, which contained more than 500 cancerous samples of papillary thyroid cancer, 59 normal samples, 8 lymph node metastatic (LNM) samples and corresponding clinical data. We performed survival analysis to identify prognostic influence, enrichment analysis to reveal relevant pathways and ontology of gene sets and miRNA target prediction to depict possible miRNA-mRNA interactions in WDTC (Fig. 1).

Patient and sample preparation. The present study was approved by the Institutional Review Board and Ethics Committee of The Shanghai Tenth People's Hospital, Tongji University, Shanghai, China (SHSY-IEC-KY-4.0/17-13/01). In March 2010, case WDTC01 was a 40-year-old male patient, diagnosed with follicular thyroid cancer and distant bone metastasis (BM) in the 11th thoracic vertebrae. Total thyroidectomy was performed at Zhongshan Hospital, Shanghai, China. Paracancerous and cancerous tissues were collected during surgery. To relieve neurological symptoms of the lower extremity caused by BM, the metastatic lesion was debulked, collected and internally fixed. After three ${ }^{131} \mathrm{I}$ radiation ablations, local recurrence was observed at the 11th thoracic vertebrae on September 2011 and the recurred lesion was collected during surgery (Fig. 1). All samples were immediately stored at $-80^{\circ} \mathrm{C}$ until use. Total RNA was harvested using TRIzol (Invitrogen; Thermo Fisher Scientific, Inc., Waltham, MA, USA) and an miRNeasy mini kit (Qiagen GmbH, Hilden, Germany) according to the manufacturer's instructions.

Genome-wide transcriptional profiling with Agilent microarray and microRNA microarray expression profiling. RNA was immediately shipped on dry ice to KangChen Bio-Tech (Shanghai, China) for mRNA and miRNA microarray assay. For analysis via the Agilent Whole Human Genome Oligo Microarray platform, total RNA from each sample was amplified and transcribed into fluorescent cRNA using the manufacturer's instructions (Agilent's Quick Amp Labeling protocol, version 5.7; Agilent Technologies, Santa Clara, CA, USA). Labeled cRNAs were hybridized onto the Whole Human Genome Oligo Microarray (4x44 K; Agilent Technologies). After washing the slides, arrays were scanned using the Agilent Scanner G2505C. Agilent Feature Extraction software (version 11.0.1.1) was used to analyze the acquired array images. Quantile normalization and subsequent data processing were performed using the GeneSpring GX v11.5.1 software package (Agilent Technologies).

For the miRNA microarray, samples were labeled using the miRCURY Hy3/Hy5 Power labeling kit and hybridized with a miRCURY LNA Array (v.16.0) (Exiqon, Vedbaek, Denmark). Following washing, the slides were scanned using the Axon GenePix 4000B microarray scanner. Scanned images were then imported into GenePix Pro 6.0 software (Axon Instruments; Molecular Devices, LLC, Sunnyvale, CA, USA) for grid alignment and data extraction. Replicated miRNAs were averaged and miRNAs in which intensities were $\geq 50$ in all samples were chosen for calculating a normalization factor.

Targeting prediction and enrichment analysis. The online prediction tool MiRWalk 2.0 (24) was used to predict interactions between miRNAs and mRNAs. In addition, miRanda (25) and TargetScan (26) were selected to generate an intersecting gene list. When an mRNA was predicted by all three databases, it was considered a target for the miRNA. Biological pathways defined by the Kyoto Encyclopedia of Genes and Genomes (KEGG) (27) and Gene Ontology (GO) (28) were analyzed using the Database for Annotation, Visualization and Integrated Discovery (DAVID) (29). DAVID provides a set of functional annotations for many genes. In the present study, KEGG pathway and GO terms were selected with a threshold of $\mathrm{P}<0.05$.

Statistical analysis. For WDTC01, mRNAs and miRNAs with fold-changes (FC) >1.5 times were defined as differentially expressed and considered for analysis. RNA sequencing data with 505 samples of papillary thyroid cancer (PTC), 59 normal control and eight LNM, with the -3 miRNA sequencing data of 502 samples of PTC, 59 normal control and eight LNM from TCGA thyroid carcinoma (THCA), and survival data were downloaded from the TCGA analytical tool UCSC Xena (http://xena.ucsc.edu). However, recurrent sites were not available. The unit for the mRNA sequencing data was 


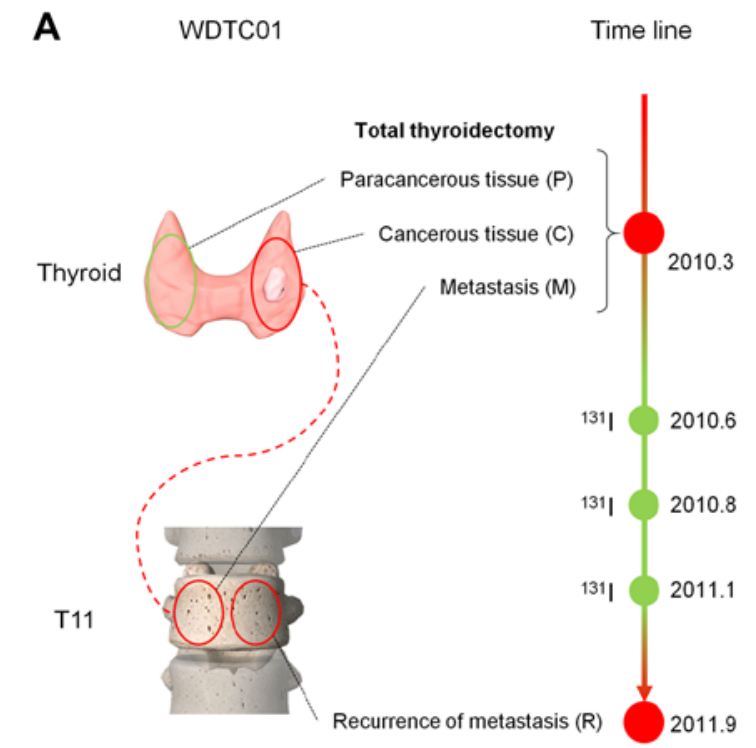

B Tumorigenesis Metastasis Progression Recurrence

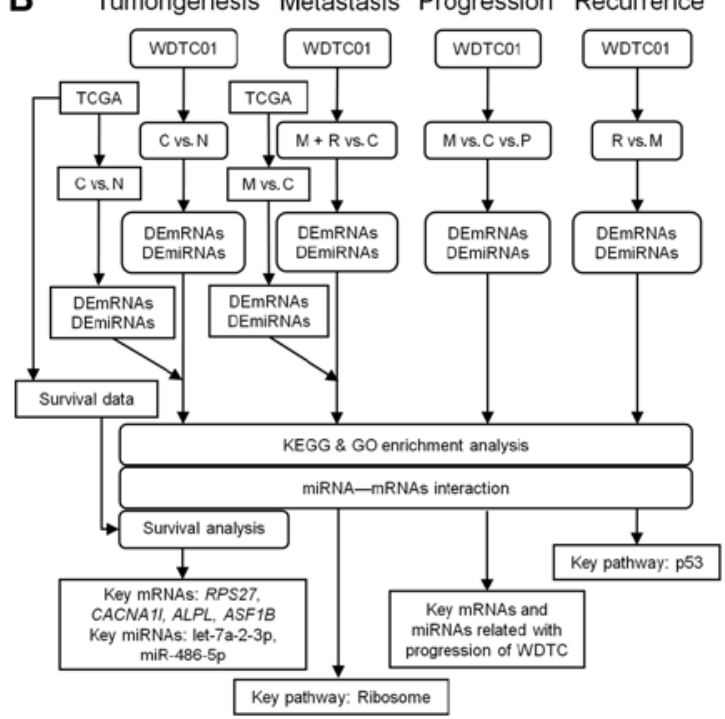

Figure 1. Schematic of sample collection and research procedure. (A) Schematic of treatment time-points and sample collections for case WDTC01. (B) Pipeline diagram represents the study work flow to observe changes in mRNA and miRNA expression across different stages of WDTC and assessment using enrichment analysis and targeting predictions. TCGA data and corresponding survival data were used to investigate tumorigenic and metastatic processes during WDTC. ${ }^{131}$ I, iodine-131 radiotherapy; T11, 11th thoracic vertebrae; WDTC, well-differentiated thyroid cancer.

$\log 2(\mathrm{RSEM}+1)$ and the unit for the miRNA sequencing data was $\log 2(\mathrm{RPM}+1)$. To screen differentially expressed mRNAs and miRNAs with a median expression $>0$, we used the linear model of the limma package (30) of R-3.4.1. Benjamin and Hochberg's (31) correction was applied to ensure a false discovery rate (FDR). For comparisons between cancer and normal samples, DEmRNAs and DEmiRNAs were identified when FC $>1.5, \mathrm{P}<0.05$ and FDR $<0.05$. For comparisons between $8 \mathrm{LNM}$ and cancer samples, criteria were $\mathrm{FC}>1.5$ and $\mathrm{P}<0.05$.

To depict expression differences, a scatter plot was used to describe averages and standard deviations of expression. To address overall survival (OS) and recurrence-free survival (RFS) for patients, we retrieved follow-up states for patients in the clinical data matrix from TCGA and merged this with the expression matrix and performed Kaplan-Meier analysis for each mRNA and miRNA with survival of R. OS and RFS were considered significant when log-rank test was $\mathrm{P}<0.05$.

\section{Results}

Upregulated ASF1B and ALPL and downregulated RPS27, CACNA1I, let-7a-2-3p and miR-486-5p are related to the prognosis of WDTC. We observed 444 upregulation and 740 downregulated DEmRNAs and 114 upregulation and 105 downregulated DEmiRNAs in cancerous tissue for WDTC01 (Fig. 2). To validate commonly expressed DEmRNAs and miRNAs in WDTC, DEmRNAs and miRNAs were compared with TCGA data. We identified 49 upregulated and 53 downregulated DEmRNAs, along with 2 upregulated and 10 downregulated DEmiRNAs, which were commonly deregulated (Fig. 3A). We selected overlapping molecules to perform survival analysis and construct a miRNA-mRNA network using online miRNA-target predicting tools. We found 10 overlapping mRNAs that interacted with 21 commonly deregulated mRNAs (Fig. 3B).
Among commonly expressed DEmRNAs and miRNAs, high expression of alkaline phosphatase, liver/bone/kidney (ALPL), low expression of ribosomal protein S27 (RPS27) and calcium voltage-gated channel subunit alpha1 I (CACNAII) were associated with overall survival of WCDT. High expression of anti-silencing function 1B histone chaperone $(A S F 1 B)$ and low expression of let-7a-2-3p and miR-486-3p were associated with RFS for WDTC (Fig. 3C).

Expression patterns are related to metastatic variations of WDTC. We identified 632 upregulation and 316 downregulated DEmRNAs and 20 upregulation and 46 downregulated DEmiRNAs in DM and recurrent lesions of WDTC01. To identify evolutionary signatures related to metastasis of WDTC, we investigated common expression patterns shared with BM from WDTC01 and LNM from TCGA. DEmRNAs and miRNAs from both metastatic sources were different. We found 18 upregulated DEmRNAs, such as apolipoprotein $\mathrm{E}(\mathrm{ApoE})$, cathepsin $\mathrm{H}(\mathrm{CTSH}), \mathrm{C}-\mathrm{X}-\mathrm{C}$ motif chemokine receptor 4 (CXCR4) and mucin 4 (MUC4) and one downregulated DEmRNA, gonadotropin releasing hormone receptor $(G N R H R)$ in BM and LNM. For miRNAs, downregulation of miR-30a-5p occurred in BM and LNM (Fig. 4A).

Hyper-activation of the ribosome pathway contributes to DM of WDTC as indicated by enrichment analysis. To analyze features of BM and LNM of WDTC, we performed enrichment analysis with DEmRNAs that did not overlap between the two metastatic sources. Ribosomes, first listed in KEGG analysis and cell component (CC) in the GO analysis, were involved.

Significant biological process (BP) and molecular function (MF) terms were related to the translational process. Our analysis focused on the ribosome pathway in which 63 enriched genes were upregulated (Fig. 4B). For LNM, KEGG terms were related to immune-related pathways. BP terms 

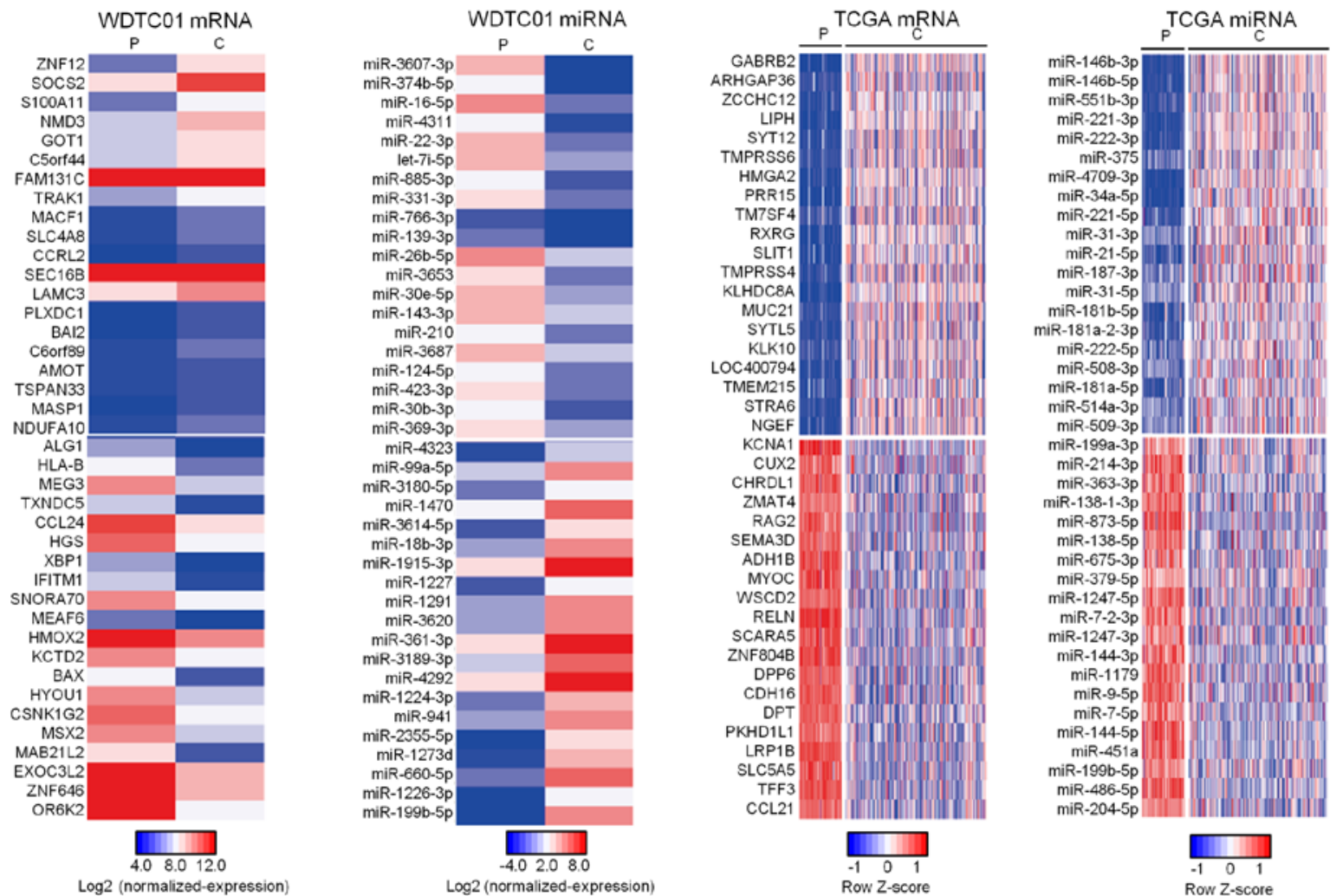

Figure 2. Heatmap plot of DEmRNAs and DEmiRNAs from WDTC01 and TCGA sequencing data. Heatmap plot of top 20 upregulation and top 20 downregulated mRNAs and miRNAs with the greatest fold-change from microarray data of WDTC01 and TCGA sequencing data of cancerous tissue (C) and paracancerous tissue $(\mathrm{P})$.
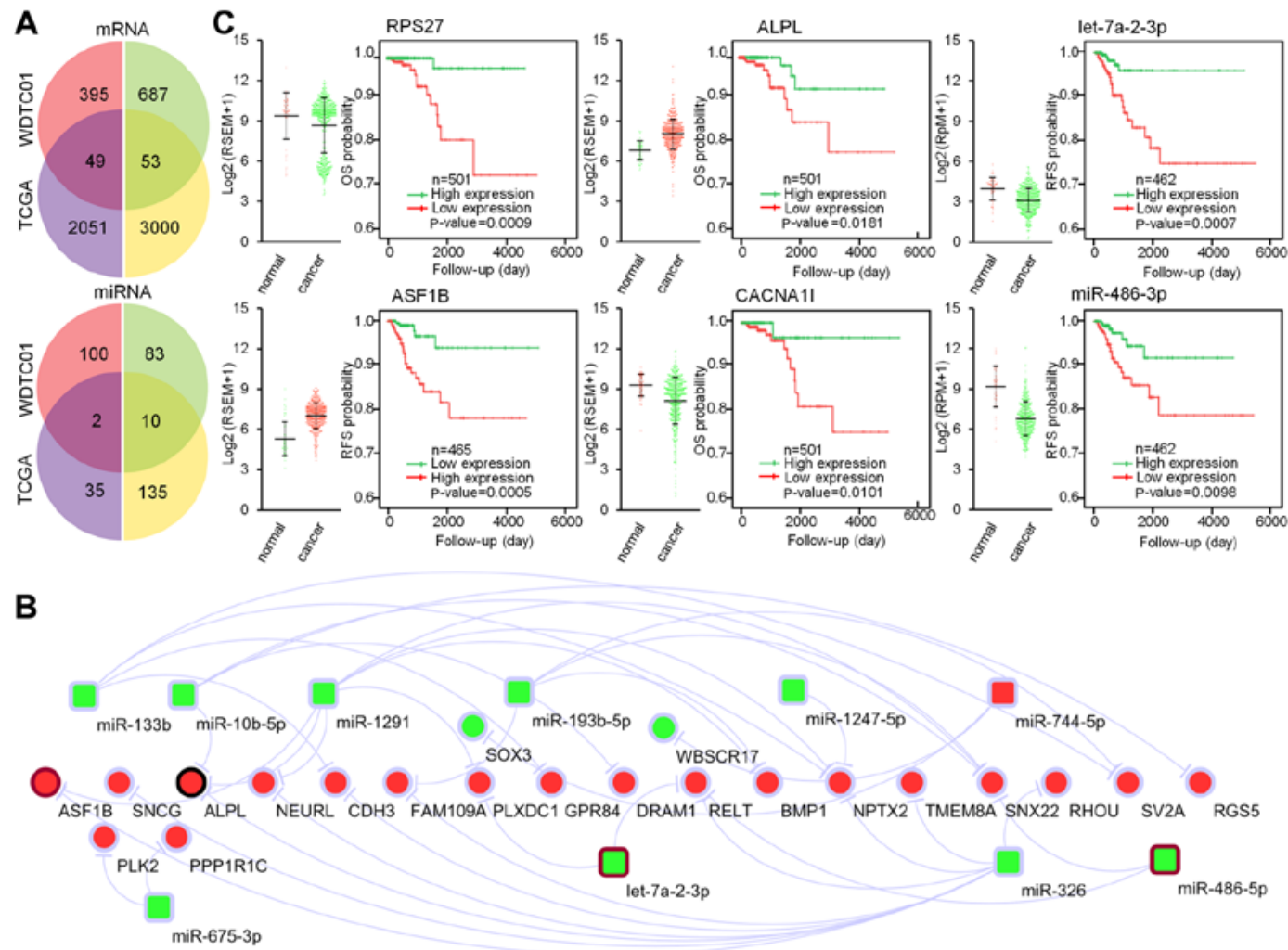

Figure 3. DEmRNAs and DEmiRNAs associated with carcinogenesis of WDTC. (A) Venn diagram of DEmRNAs and DEmiRNAs from WDTC01(red, upregulation; green, downregulation) overlapped with TCGA data (purple, upregulation; yellow, downregulation) (B) Using overlapping DEmRNAs and DEmiRNAs, a miRNA-mRNA interaction network was created by applying three target predicting algorithms. Nodes with black borders were related to OS and nodes with brown borders were related to RFS. (C) Expression and Kaplan-Meier curves of four overlapped mRNAs and two overlapped miRNAs associated with OS or RFS. 

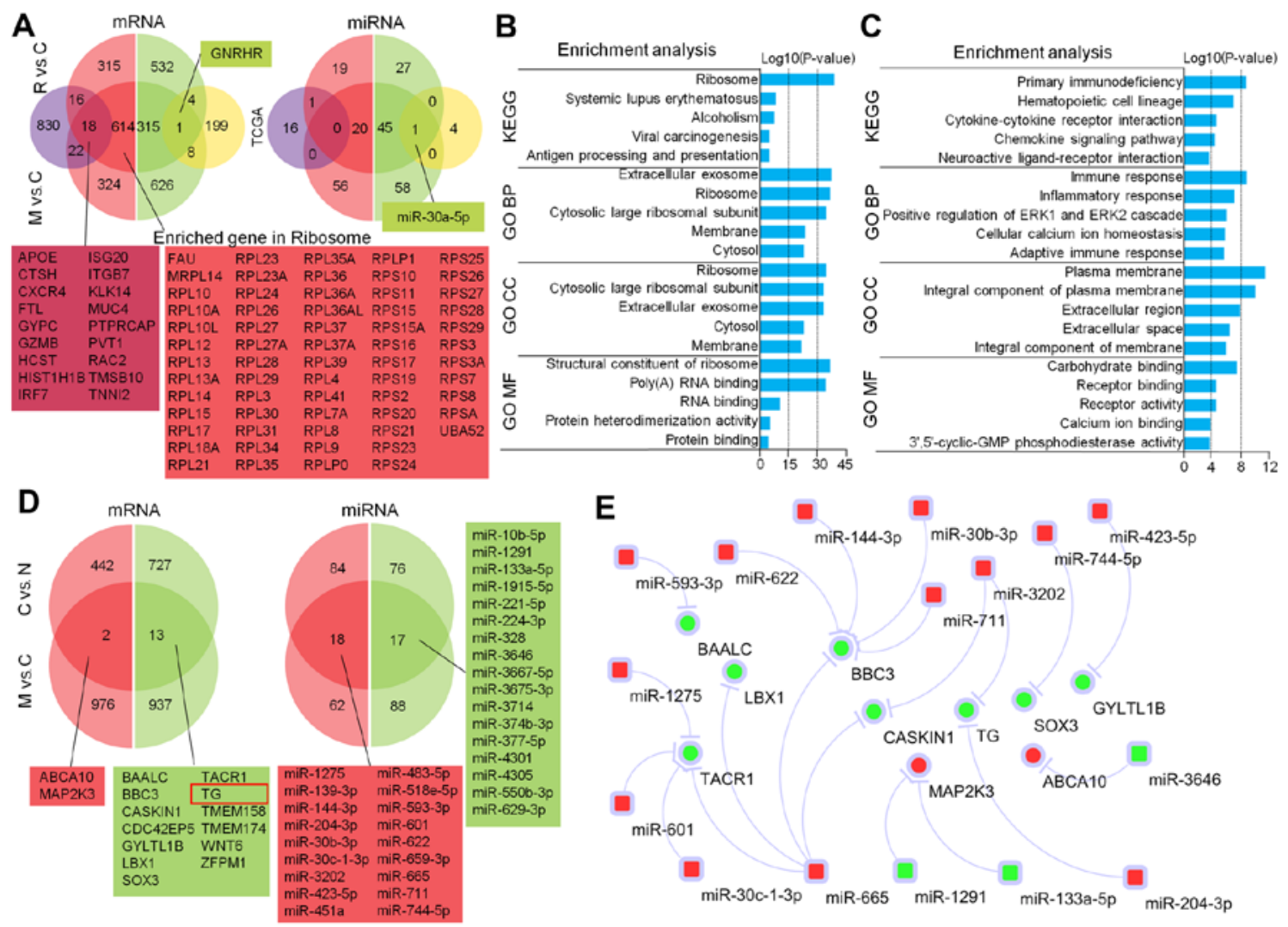

Figure 4. DEmRNAs and DEmiRNAs associated with metastasis of WDTC. (A) Venn diagram of DEmRNAs and DEmiRNAs from WDTC01, featuring expression changes in metastatic lesions (M) and local metastatic recurrence (R) compared to cancerous tissue (C) (red, upregulation; green, downregulation) overlapped with eight LMN from TCGA data (purple, upregulation; yellow, downregulation). All genes enriched in the ribosome pathway were upregulated (red squares) (B) KEGG and GO enrichment analysis of 614 upregulated and 315 downregulated mRNA specified in BM shows a strong relationship with translational activity. (C) KEGG and GO enrichment analysis of 830 upregulated and 199 downregulated mRNA specified in the LMN lesion revealed an association with the cytokine receptor and the chemokine signaling pathway. (D) Overlapped mRNAs and miRNAs in Venn diagram represent upregulation and downregulated genes comparing normal tissue (N) to cancerous tissue to metastasis. TG, marked by a red box, continuously downregulated in WDTC01. (E) Putative regulatory network regarding overlapped mRNAs and miRNAs.

were related to the ERK1/ERK2 signaling pathway and cellular calcium ion homeostasis. CC terms were related to extracellular activity and MF terms were associated with regulation of ion and protein binding (Fig. 4C).

Comparison among different stages of WDTC reveals that upregulation and downregulated DEmRNAs and DEmiRNAs are associated with tumor progression. To investigate DEmRNAs and miRNAs associated with tumor progression, we analyzed three stages of WDTC01 (normal, cancerous and metastatic tissues) before ${ }^{131}$ I treatment. Upregulated DEmRNAs included ATP-binding cassette subfamily A member 10 (ABCA10) and Mitogen-Activated Protein Kinase Kinase 3 (MAP2K3) and 13 downregulated DEmRNAs, such as BCL2 binding component 3 (BBC3) and thyroglobulin $(T G)$, as well as 18 upregulation and 17 downregulated DEmiRNAs that were constantly deregulated with tumor progression (Fig. 4D).

Potential interactions among DEmRNAs and miRNAs using the aforementioned algorithm suggested that downregulation of miR-1291, miR-133a-5p and miR-3646 may increase the expression of $M A P 2 K 3$ and $A B C A 10$. Downregulation of the pro-apoptotic gene $B B C 3$ may be caused by increases in 5 different progression-related miRNAs. The lineage upregulation of miR-204-3p and miR-3202 may interfere with the expression of $T G$, an important marker for thyroid cancer cell stemness (Fig. 4E).

Regained TG expression and impaired p53 downstream in recurrent lesions. To understand the underlying molecular mechanisms for recurrence following three ${ }^{131}$ I treatments, we compared recurrence and metastasis and revealed that there were 740 upregulation and 754 downregulated mRNAs and 88 upregulation and 60 downregulated miRNAs. $T G$ was the most upregulated gene (10-fold) in recurrent lesions. Pro-apoptotic genes $B A X(\mathrm{FC}=23.6)$ and caspase- $8(\mathrm{FC}=9.2)$ were among the top 20 downregulated genes (Fig. 5A).

KEGG pathway enrichment analysis was applied to 1,494 DEmRNAs and the p53 signaling pathway was of interest (Fig. 5B). Downregulation of BCL2 associated X, apoptosis regulator $(B A X)$ and caspase-8 $(C A S P 8)$ and upregulation of cyclin B1 (CCB1), D1 (CCDI) and E1 (CCE1) indicated attenuated downstream effects of the p53 pathway, such as cell cycle arrest and apoptosis (Fig. 5C). MiRNA-mRNA interaction analysis revealed potential DEmiRNAs in recurrent lesions, which regulated these mRNAs downstream of the p53 pathway. Among apoptotic-inducing genes, $B A X$ and $C A S P 8$ were targeted by several miRNAs, and miR-1827 interacted with $B A X$ and CASP8; EI24 was targeted by miR-211-5p, a well-known oncogene in WDTC (Fig. 5D). 
A

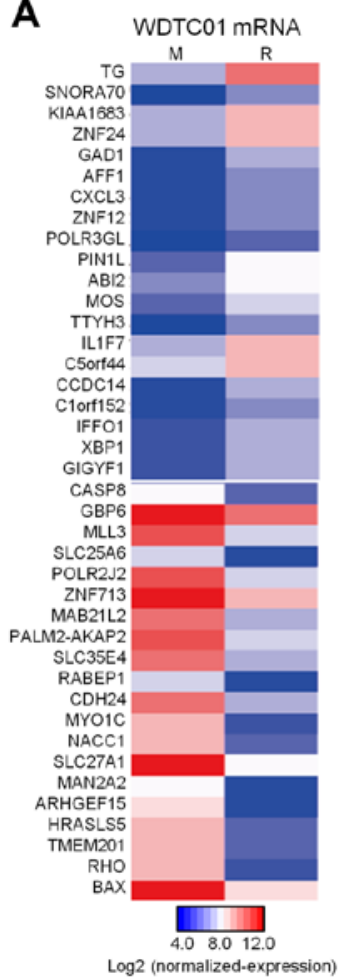

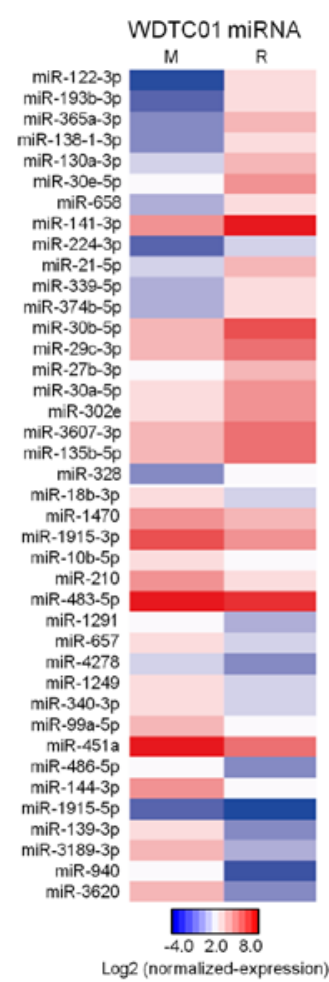

B

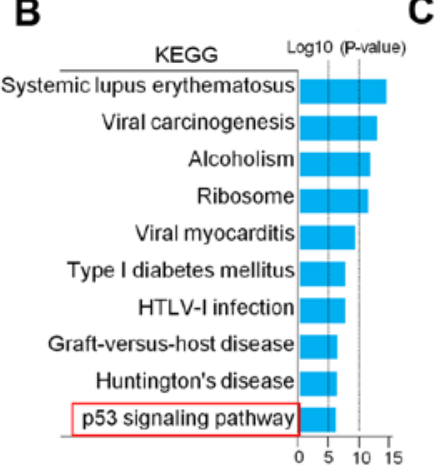

C Genesin

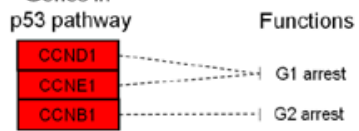

D

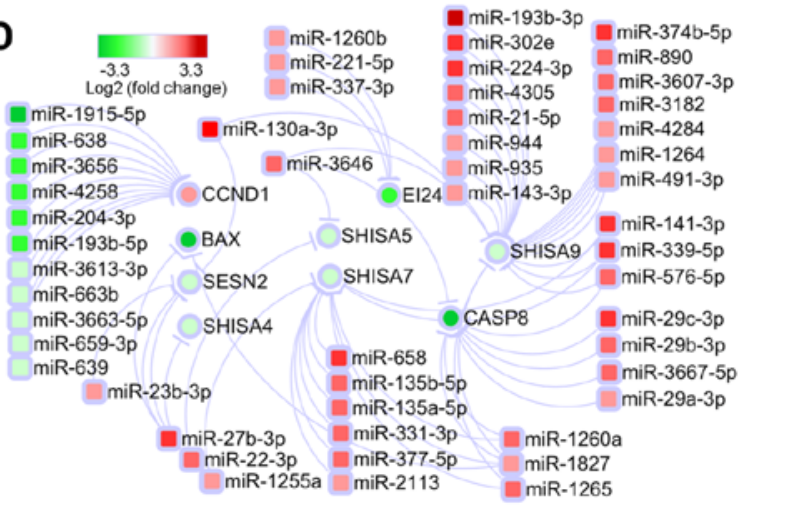

Figure 5. DEmRNAs and DEmiRNAs associated with recurrence of WDTC. (A) Heatmap plot of top 20 upregulation and top 20 downregulated mRNAs and miRNAs with the greatest fold-change regarding expression alterations after ${ }^{131} \mathrm{I}$ treatment. (B) Top 10 KEGG pathway enrichment analysis of DEmRNAs with the greatest $\log _{10}$ (P-value). (C) DEmRNAs enriched in the p53 pathway revealed impaired downstream function of the p53 pathway in cancer recurrence. Red box, upregulated genes; green box, downregulated genes. (D) Regulatory network of deregulated genes in the p53 pathway. Node colors reflect fold-changes.

\section{Discussion}

Despite increasing WDTC globally, few studies have investigated metastatic sites of WDTC from a genomic perspective. RNA-Seq analysis of metastatic sites, including two LNM and one pleural metastasis from a single patient, revealed intratumor heterogeneity and clonal evolution (32). Another miRNA study analyzed miRNA transcriptomes of PTC with four LNM and noted that downregulation of miRNAs was a common feature in PTC tumorigenesis (33). However, there has been no study to investigate the underlying mechanisms of recurrent WDTC using recurrent lesions and genomic profiling due to the difficulty of collecting tissue. We used one paired bone metastatic and one paired recurrent metastatic lesion and performed mRNA and miRNA microarray analysis. Integrated with TCGA data, we found common prognostic factors as key expression changes related to metastasis and distinct features of LMN and BM. We also suggested a pattern for recurrence from a genomic perspective, and we provided evidence for future prevention and therapy for metastasis and relapse of WDTC.

The epithelial-mesenchymal transition is a well-established model explaining thyroid cancer development (34). $T G$ is thought to be a differentiation marker for WDTC (35), and lower $T G$ expression was revealed to be correlated with thyroid cancer cell stemness (34). For WDTC01, $T G$ expression gradually decreased in normal tissue to metastatic stage tissue (Fig. 4D). This may be explained by the fact that tumor cells with lower $T G$ expression migrating from the primary tumor are prone to BM, which is supported by the fact that poorly differentiated thyroid cancer has more DM (36). Additionally, significant repression of $T G$ was absent in 8 LNM from TCGA, indicating that $T G$ suppression is specifically related to BM and not LNM. Next, the microenvironment of the vertebrae may induce an adaptive response to suppress expression of $T G$. According to the miRNA-mRNA analysis, TG may be suppressed by aberrant upregulation of miR-3202 and miR-204-3p (Fig. 4D). Along with $T G$, the pro-apoptotic $B B C 3$ gene was gradually downregulated during lineage dedifferentiation. The putative miRNA-mRNA network revealed that continuously increasing expression of miR-622, miR-144-3p, miR-30b-3p, miR-711 and miR-665 may be the trigger of dysregulation of $B B C 3$.

High expression of $A L P L$ and low expression of miR-486-5p were related to a prognosis of WDTC, and these markers were associated with cancer cell stemness. High expression of $A L P L$, as a pluripotent stem cell marker (37), has been revealed to be associated with poor OS for glioblastoma and prostate cancers $(38,39)$. For lung and prostate cancer, downregulation of miR-486-5p was reported to be associated with poor survival (40-42). Cellular function data revealed that loss of miR-486-5p dedifferentiated cancer cells via epithelial-mesenchymal transition (42).

To find the common molecules related to BM and LNM in WDTC, we overlapped expression changes in both and among the 19 overlapped DEmRNAs, and CXCR4 was key to the $C X C L 12 / C X C R 4$ pathway, a critical and well-studied pathway in BM (43). CXCR4 was reported to be significantly upregulated and related to BRAF mutations and neoplastic infiltration, resulting in more aggressive WDTC (44). Another 
two molecules involved in WDTC progression were also related to cancer cell stemness. As reported in ovarian carcinoma (45), MUC4 can induce epithelial-mesenchymal transition, and it can enhance the invasion of tumors in pancreatic and breast cancers $(46,47)$. miR-30a-5p, the only overlapped miRNA in metastatic lesions, is reported to be associated with cancer metastasis. The loss of miR-30a-5p can lead to invasion and migration of breast cancer (48) and colorectal cancer cells (49) and epithelial-mesenchymal transition (50).

In addition, BM and LNM had distinct features with respect to enrichment analysis. Notably, all KEGG and GO terms of BM for WDTC01 were associated with ribosomal activity, and all genes enriched in this pathway were upregulated, indicating that efficient ribosome translational machineries contribute to $\mathrm{BM}$ in WDCT01. Although individual ribosomal proteins, such as RPL17 and RPS27L, were reported to be tumor suppressors, ribosome biogenesis and translation were directly associated with increased cell growth and proliferation (51). In contrast, no sign of overturned ribosomal activity was observed in the 8 LNM, perhaps due to the different microenvironment between BM and LNM.

The most common reason for WDTC recurrence was due to poor uptake of ${ }^{131} \mathrm{I}$ in the poorly differentiated variant of WDTC. For WDTC01, gradually decreased expression of TG indicated a more dedifferentiated type of WDTC, so inefficient uptake was considered to contribute to cancer relapse (52). However, following ${ }^{131} \mathrm{I}$ treatments, expression differed greatly from former metastatic lesions, indicating heterogeneity of the recurrent lesion. Well-differentiated neoplasms arise from residual cancer stem cells (CSCs) following surgical debulking. $T G$ expression was again recovered to its pre-metastatic state because CSCs give rise to differentiated progeny (53). According to enrichment analysis, several critical genes of the p53 pathway were altered, and apoptotic resistance and a promoted cell cycle should increase proliferation of the recurrent lesion.

In summary, we offered an integrated analysis of mRNAs and miRNAs involved in the metastasis, progression and recurrence of WDTC and identified numerous mRNA and miRNA related to stemness, which contributed to the malignancy and recurrence of WDTC. The ribosome pathway was hyper-activated in BM, and the p53 pathway was correlated with relapse in our case. This information may provide useful regulatory targets and pathways for future development of predictive tools and therapies of WDTC.

\section{Acknowledgements}

We thank the study subject for his participation. We appreciate the experimental support of the Central Laboratory for Medical Research, Shanghai Tenth People's Hospital.

\section{Funding}

The present study was supported partly by grants from the National Natural Science Foundation of China (81472202, 81772932,81201535,81302065,81301993,81472501,81372175, 81472209 and 81702243), The Fundamental Research Funds For the Central Universities (22120170212 and 22120170117), The Shanghai Natural Science Foundation (12ZR1436000 and 16ZR1428900) and the Shanghai Municipal Commission of Health and Family Planning (201440398 and 201540228). The funder has no role in the study design, data collection and analysis, decision to publish or preparation of the manuscript.

\section{Availability of data and materials}

The datasets used during the present study are available from the corresponding author upon reasonable request.

\section{Authors' contributions}

YJZ, YSM, QX, DF and XFW designed the study. YJZ, YSM, QX, FY, ZWL, CYJ, DF and XFW performed the experiments. YJZ, YSM, DF and XFW performed the statistical analyses and interpreted the data. QX, FY, ZWL, CYJ, GXL, $\mathrm{XQY}$ and $\mathrm{XFW}$ are involved in the patient recruitment. FY, ZWL, CYJ, XXJ, LZ, YCS, WTX, GXL, XQY, PZ, DF and XFW contributed to the study materials and consumables. YJZ, YSM, DF and XFW wrote the manuscript. YJZ, YSM and QX contributed equally to this work. All authors read and approved the manuscript and agree to be accountable for all aspects of the research in ensuring that the accuracy or integrity of any part of the work are appropriately investigated and resolved.

\section{Ethics approval and consent to participate}

The present study was approved by the Institutional Review Board and Ethics Committee of The Shanghai Tenth People's Hospital, Tongji University, Shanghai, China (SHSY-IEC-KY-4.0/17-13/01).

\section{Patient consent for publication}

Not applicable.

\section{Competing interests}

The authors declare that they have no competing interests.

\section{References}

1. Kilfoy BA, Zheng T, Holford TR, Han X, Ward MH, Sjodin A, Zhang Y, Bai Y, Zhu C, Guo GL, et al: International patterns and trends in thyroid cancer incidence, 1973-2002. Cancer Causes Control 20: 525-531, 2009.

2. Chen AY, Jemal A and Ward EM: Increasing incidence of differentiated thyroid cancer in the United States, 1988-2005. Cancer 115: 3801-3807, 2009.

3. Sherman SI: Thyroid carcinoma. Lancet 361: 501-511, 2003.

4. Xing M, Haugen BR and Schlumberger M: Progress in molecular-based management of differentiated thyroid cancer. Lancet 381: 1058-1069, 2013.

5. Kimura ET, Nikiforova MN, Zhu Z, Knauf JA, Nikiforov YE and Fagin JA: High prevalence of $B R A F$ mutations in thyroid cancer: Genetic evidence for constitutive activation of the RET/ PTC-RAS-BRAF signaling pathway in papillary thyroid carcinoma. Cancer Res 63: 1454-1457, 2003.

6. Cohen Y, Xing M, Mambo E, Guo Z, Wu G, Trink B, Beller U, Westra WH, Ladenson PW and Sidransky D: BRAF mutation in papillary thyroid carcinoma. J Natl Cancer Inst 95: 625-627, 2003. 
7. Ciampi R, Knauf JA, Kerler R, Gandhi M,Zhu Z, Nikiforova MN, Rabes HM, Fagin JA and Nikiforov YE: Oncogenic AKAP9$B R A F$ fusion is a novel mechanism of MAPK pathway activation in thyroid cancer. J Clin Invest 115: 94-101, 2005.

8. Wreesmann VB, Sieczka EM, Socci ND, Hezel M, Belbin TJ, Childs G, Patel SG, Patel KN, Tallini G, Prystowsky M, et al: Genome-wide profiling of papillary thyroid cancer identifies MUC1 as an independent prognostic marker. Cancer Res 64: 3780-3789, 2004.

9. Moon S, Song YS, Kim YA, Lim JA, Cho SW, Moon JH, Hahn S, Park DJ and Park YJ: Effects of coexistent BRAF ${ }^{\mathrm{V} 600 \mathrm{E}}$ and TERT promoter mutations on poor clinical outcomes in papillary thyroid cancer: A meta-analysis. Thyroid 27: 651-660, 2017.

10. Lee JJ, Wang TY, Liu CL, Chien MN, Chen MJ, Hsu YC, Leung $\mathrm{CH}$ and Cheng SP: Dipeptidyl peptidase IV as a prognostic marker and therapeutic target in papillary thyroid carcinoma. J Clin Endocrinol Metab 102: 2930-2940, 2017.

11. Chowdhury S, Veyhl J, Jessa F, Polyakova O, Alenzi A, MacMillan C, Ralhan R and Walfish PG: Programmed death-ligand 1 overexpression is a prognostic marker for aggressive papillary thyroid cancer and its variants. Oncotarget 7: 32318-32328, 2016.

12. Samaan NA, Schultz PN, Hickey RC, Goepfert H, Haynie TP, Johnston DA and Ordonez NG: The results of various modalities of treatment of well differentiated thyroid carcinomas: A retrospective review of 1599 patients. J Clin Endocrinol Metab 75: 714-720, 1992

13. Schlumberger MJ: Papillary and follicular thyroid carcinoma. $\mathrm{N}$ Engl J Med 338: 297-306, 1998.

14. Durante C,Haddy N, Baudin E, Leboulleux S, Hartl D, Travagli JP, Caillou B, Ricard M, Lumbroso JD, De Vathaire F, et al: Long-term outcome of 444 patients with distant metastases from papillary and follicular thyroid carcinoma: Benefits and limits of radioiodine therapy. J Clin Endocrinol Metab 91: 2892-2899, 2006.

15. Haugen BR: 2015 American Thyroid Association management guidelines for adult patients with thyroid nodules and differentiated thyroid cancer: What is new and what has changed? Cancer 123: 372-381, 2017.

16. O'Neill CJ, Oucharek J, Learoyd D and Sidhu SB: Standard and emerging therapies for metastatic differentiated thyroid cancer. Oncologist 15: 146-156, 2010.

17. Mazzaferri EL and Kloos RT: Clinical review 128: Current approaches to primary therapy for papillary and follicular thyroid cancer. J Clin Endocrinol Metab 86: 1447-1463, 2001.

18. Mazzaferri EL and Jhiang SM: Differentiated thyroid cancer long-term impact of initial therapy. Trans Am Clin Climatol Assoc 106: 151-168; discussion 168-170, 1995.

19. Visone R, Russo L, Pallante P, De Martino I, Ferraro A, Leone V, Borbone E, Petrocca F, Alder H, Croce CM and Fusco A: MicroRNAs (miR)-221 and miR-222, both overexpressed in human thyroid papillary carcinomas, regulate $\mathrm{p} 27 \mathrm{Kip} 1$ protein levels and cell cycle. Endocr Relat Cancer 14: 791-798, 2007.

20. Mancikova V, Castelblanco E, Pineiro-Yanez E, Perales-Paton J, de Cubas AA, Inglada-Perez L, Matias-Guiu X, Capel I, Bella M, Lerma E, et al: MicroRNA deep-sequencing reveals master regulators of follicular and papillary thyroid tumors. Mod Pathol 28: 748-757, 2015

21. Cancer Genome Atlas Research Network: Integrated genomic characterization of papillary thyroid carcinoma. Cell 159 676-690, 2014

22. Zhao M, Wang KJ, Tan Z, Zheng CM, Liang Z and Zhao JQ: Identification of potential therapeutic targets for papillary thyroid carcinoma by bioinformatics analysis. Oncol Lett 11 : 51-58, 2016.

23. Geraldo MV and Kimura ET: Integrated analysis of thyroid cancer public datasets reveals role of post-transcriptional regulation on tumor progression by targeting of immune system mediators. PLoS One 10: e0141726, 2015.

24. Dweep H and Gretz N: miRWalk2.0: A comprehensive atlas of microRNA-target interactions. Nat Methods 12: 697, 2015.

25. Betel D, Wilson M, Gabow A, Marks DS and Sander C: The microRNA.org resource: Targets and expression. Nucleic Acids Res 36: D149-D153, 2008.

26. Agarwal V, Bell GW, Nam JW and Bartel DP: Predicting effective microRNA target sites in mammalian mRNAs. Elife 4: e05005, 2015.
27. Kanehisa M, Sato Y, Kawashima M, Furumichi M and Tanabe M: KEGG as a reference resource for gene and protein annotation. Nucleic Acids Res 44: D457-D462, 2016.

28. Ashburner M, Ball CA, Blake JA, Botstein D, Butler H, Cherry JM, Davis AP, Dolinski K, Dwight SS, Eppig JT, et al: Gene ontology: Tool for the unification of biology. The gene ontology consortium. Nat Genet 25: 25-29, 2000.

29. Huang da W, Sherman BT and Lempicki RA: Systematic and integrative analysis of large gene lists using david bioinformatics resources. Nat Protoc 4: 44-57, 2009.

30. Ritchie ME, Phipson B, Wu D, Hu Y, Law CW, Shi W and Smyth GK: Limma powers differential expression analyses for rna-sequencing and microarray studies. Nucleic Acids Res 43: e47, 2015

31. Benjamini $\mathrm{Y}$ and Hochberg $\mathrm{Y}$ : Controlling the false discovery rate-a practical and powerful approach to multiple testing. J R Stat Soc Ser B-Methodol 57: 289-300, 1995.

32. Le Pennec S, Konopka T, Gacquer D, Fimereli D, Tarabichi M, Tomas G, Savagner F, Decaussin-Petrucci M, Tresallet C, Andry G, et al: Intratumor heterogeneity and clonal evolution in an aggressive papillary thyroid cancer and matched metastases. Endocr Relat Cancer 22: 205-216, 2015.

33. Saiselet M, Gacquer D, Spinette A, Craciun L, DecaussinPetrucci M, Andry G, Detours V and Maenhaut C: New global analysis of the microRNA transcriptome of primary tumors and lymph node metastases of papillary thyroid cancer. BMC Genomics 16: 828, 2015.

34. Lin RY: Thyroid cancer stem cells. Nat Rev Endocrinol 7: 609-616, 2011.

35. Takano T, Miyauchi A, Yokozawa T, Matsuzuka F, Maeda I, Kuma K and Amino N: Preoperative diagnosis of thyroid papillary and anaplastic carcinomas by real-time quantitative reverse transcription-polymerase chain reaction of oncofetal fibronectin messenger RNA. Cancer Res 59: 4542-4545, 1999.

36. Are $C$ and Shaha AR: Anaplastic thyroid carcinoma: Biology, pathogenesis, prognostic factors, and treatment approaches. Ann Surg Oncol 13: 453-464, 2006.

37. Hou Z, Meyer S, Propson NE, Nie J, Jiang P, Stewart R and Thomson JA: Characterization and target identification of a DNA aptamer that labels pluripotent stem cells. Cell Res 25: 390-393, 2015.

38. Rao SR, Snaith AE, Marino D, Cheng X, Lwin ST, Orriss IR, Hamdy FC and Edwards CM: Tumour-derived alkaline phosphatase regulates tumour growth, epithelial plasticity and disease-free survival in metastatic prostate cancer. $\mathrm{Br} \mathbf{J}$ Cancer 116: 227-236, 2017.

39. Iwadate Y, Suganami A, Tamura Y, Matsutani T, Hirono S, Shinozaki N, Hiwasa T, Takiguchi M and Saeki N: The pluripotent stem-cell marker alkaline phosphatase is highly expressed in refractory glioblastoma with DNA hypomethylation. Neurosurgery 80: 248-256, 2017.

40. Vosa U, Vooder T, Kolde R, Vilo J, Metspalu A and Annilo T: Meta-analysis of microRNA expression in lung cancer. Int $\mathrm{J}$ Cancer 132: 2884-2893, 2013.

41. Tan X, Qin W, Zhang L, Hang J, Li B, Zhang C, Wan J, Zhou F, Shao K, Sun Y, et al: A 5-microRNA signature for lung squamous cell carcinoma diagnosis and hsa-miR-31 for prognosis. Clin Cancer Res 17: 6802-6811, 2011.

42. Zhang X, Zhang T, Yang K, Zhang M and Wang K: miR-486-5p suppresses prostate cancer metastasis by targeting Snail and regulating epithelial-mesenchymal transition. Onco Targets Ther 9: 6909-6914, 2016.

43. Burton A: Regulation of RANKL might reduce bone metastases. Lancet Oncol 7: 367, 2006.

44. Torregrossa L, Giannini R, Borrelli N, Sensi E, Melillo RM, Leocata P, Materazzi G, Miccoli P, Santoro M and Basolo F: Cxcr4 expression correlates with the degree of tumor infiltration and braf status in papillary thyroid carcinomas. Mod Pathol 25: 46-55, 2012.

45. Ponnusamy MP, Lakshmanan I, Jain M, Das S, Chakraborty S, Dey P and Batra SK: Muc4 mucin-induced epithelial to mesenchymal transition: A novel mechanism for metastasis of human ovarian cancer cells. Oncogene 29: 5741-5754, 2010.

46. Singh AP, Moniaux N, Chauhan SC, Meza JL and Batra SK: Inhibition of muc4 expression suppresses pancreatic tumor cell growth and metastasis. Cancer Res 64: 622-630, 2004. 
47. Rowson-Hodel AR, Wald JH, Hatakeyama J, O'Neal WK, Stonebraker JR, VanderVorst K, Saldana MJ, Borowsky AD Sweeney C and Carraway KL III: Membrane mucin muc4 promotes blood cell association with tumor cells and mediates efficient metastasis in a mouse model of breast cancer. Oncogene 37: 197-207, 2018.

48. Li W, Liu C, Zhao C, Zhai L and Lv S: Downregulation of $\beta 3$ integrin by miR-30a-5p modulates cell adhesion and invasion by interrupting Erk/Ets-1 network in triple-negative breast cancer. Int J Oncol 48: 1155-1164, 2016.

49. Wei W, Yang Y, Cai J, Cui K, Li RX, Wang H, Shang X and Wei D: miR-30a-5p suppresses tumor metastasis of human colorectal cancer by targeting ITGB3. Cell Physiol Biochem 39: 1165-1176, 2016.
50. Chung YH, Li SC, Kao YH, Luo HL, Cheng YT, Lin PR, Tai MH and Chiang PH: miR-30a-5p inhibits epithelial-to-mesenchymal transition and upregulates expression of tight junction protein claudin-5 in human upper tract urothelial carcinoma cells. Int J Mol Sci 18: E1826, 2017.

51. Ruggero D and Pandolfi PP: Does the ribosome translate cancer? Nat Rev Cancer 3: 179-192, 2003.

52. Ma ZF and Skeaff SA: Thyroglobulin as a biomarker of iodine deficiency: A review. Thyroid 24: 1195-1209, 2014

53. Vermeulen L, Sprick MR, Kemper K, Stassi G and Medema JP: Cancer stem cells-old concepts, new insights. Cell Death Differ 15: 947-958, 2008 